\title{
High-resolution R-banding and localization of fragile sites in Oryctolagus cuniculus
}

\author{
BS Poulsen, M Rønne \\ Odense University, Institute of Medical Biology, Department \\ of Anatomy and Cytology, Campusvej 55, DK-5230 Odense M, Denmark
}

(Proceedings of the 9th European Colloquium on Cytogenetics of Domestic Animals; Toulouse-Auzeville, 10-13 July 1990)

rabbit / R-banding / fragile sites / X chromosome

\section{INTRODUCTION}

Fragile sites on chromosomes are points where chromosomes are liable to break. They are divided into two major groups, common and rare, according to their population frequency and their mode of unmasking (Sutherland and Hecht, 1985; Hecht et al, 1990). In humans, fragile sites have been correlated to mental retardation (Lubs, 1968), to cancer breakpoints (De Braekeleer, 1987), to targets for mutagens and carcinogens (Yunis et al, 1987) and to breakpoints involved in the chromosomal evolution of primates (Miró et al, 1987). Yunis and Soreng (1984) found several fragile sites shared by man, chimpanzee and gorilla demonstrating that fragile sites are conserved among closely related species. Data on fragile sites in chromosomes of domesticated animals are limited, but they have been demonstrated in the pig (Riggs and Chrisman, 1989) and the horse (Rønne and Poulsen, unpublished). This paper reports on 8 putative fragile sites located in the rabbit karyotype.

\section{MATERIALS AND METHODS}

Four animals, 2 males and 2 females, were randomly selected from the stock of laboratory rabbits at the Institute of Biomedicine, Odense University. R-band induction and fragile site-unmasking were performed simultaneously as follows: peripheral blood was allowed to sediment for $1 \mathrm{~h}$ at room temperature and $0.5 \mathrm{ml}$ from the buffy/plasma layer was added to $9.5 \mathrm{ml}$ of RPMI-1640 (Gibco) supplemented with $10 \%$ fetal calf serum (Gibco), $5 \mathrm{IU} / \mathrm{ml}$ heparin (Sigma), $50 \mu \mathrm{g} / \mathrm{ml}$ gentamycin (Sigma) and phytohemagglutinin (PHA-M) (Gibco). Cultures were synchronized with fluorouracil (FU; Sigma; $5 \times 10^{-7} \mathrm{M}$ ) and processed as previously described (Rønne, 1984). For each animal, 4 cultures (1 control and 3 test cultures) were 
exposed to different chemicals as listed in table I. Subsequent harvesting, staining and band induction were performed as described by Rønne $(1983,1984)$.

Table I. Time (h) for chemical addition.

\begin{tabular}{|c|c|c|c|c|c|c|c|c|}
\hline Culture & $\begin{array}{c}F U \\
65 \mu g\end{array}$ & $\begin{array}{l}B r d U \\
0 \mu g / m\end{array}$ & $\begin{array}{l}\text { Hoechst } \\
60 \mu \mathrm{g} / \mathrm{ml}\end{array}$ & $\begin{array}{c}A P C \\
0.5 \mu \mathrm{g} / \mathrm{m}\end{array}$ & $\begin{array}{c}A Z A \\
.6 \mu \mathrm{g} / \mathrm{m}\end{array}$ & $\begin{array}{c}C A F \\
30 \mu \mathrm{g} / \mathrm{m}\end{array}$ & $\begin{array}{l}C O L \\
2 \mu g /\end{array}$ & Harvest \\
\hline 1 & 48 & 66 & 66 & & & & 70 & 72 \\
\hline 2 & 48 & 66 & 66 & $48(\mathrm{x})$ & $48(y)$ & $48(\mathrm{z})$ & 70 & 72 \\
\hline
\end{tabular}

FU: fluorouracil; BrdU: bromodeoxyuridine; Hoechst: Hoechst 33258; ACP: aphidicolin; AZA: $5^{\prime}$-cytidine; CAF: caffeine; COL: colcemid; 1: control culture; 2: test cultures $\mathrm{x}, \mathrm{y}, \mathrm{z}$.

\section{RESULTS AND DISCUSSION}

For each animal, 4 cultures ( 1 control and 3 test cultures) were examined for the presence of fragile sites. From each culture, 50 metaphases were selected at random, photographed and analyzed on a screen at a magnification of $\times 5000$. If chromosomes were difficult to identify directly, photographic copies were made and karyotyping performed.

For all 4 animals, the putative fragile sites were not expressed in the control cultures (control), while the test cultures (table I: $\mathrm{x}, \mathrm{y}, \mathrm{z}$ ) yielded metaphases with non-randomly distributed chromosomal breaks. The localization of chromosomal breaks is shown in figure 1 . The mode of action of the 3 agents employed is similar, but APC seems to be the most potent one. Only two sites, 1p32 and Xp14, were sensitive to all 3 agents. Fragility of these sites was expressed in all animals at high frequencies. In the females, Xp14 was expressed both on the active and the inactive $\mathrm{X}$-chromosome at approximately the same frequencies.

Chromosomal breaks were also found in 1q26, 4p14, 4q12, 4q14, 15q12, Xq24, but only at low frequencies (range: $2-8 \%$ ). Breakpoints at $4 \mathrm{q} 12$ and $4 \mathrm{q} 14$ were only observed in one animal (A) after APC exposure. Both females displayed a breakpoint at Xq24. This specific break was only unmasked at a relatively low frequency after APC exposure. It was only detected on the active X chromosome, but this may be due to the staining technique used or to a sampling error. In rare cases, the X chromosome displayed both breakpoints (fig 1). It is interesting that the laboratory rabbit has a breakpoint at Xq24 similar to that observed for horses and humans. The Y chromosome showed no breaks either in the controls or in the test cultures. The most important findings are summarized in table II.

According to Hecht et al (1990), the status of a fragile site may be either tentative, provisional or confirmed. Judged from the frequencies and the distribution of the breakpoints observed in the rabbit karyotype, these fall into two separate groups. The breakpoints $1 \mathrm{p} 32$ and Xp14 may represent APC-sensitive common fragile sites with a provisional status, while the other breakpoints may be either rare or common fragile sites with a tentative status. 

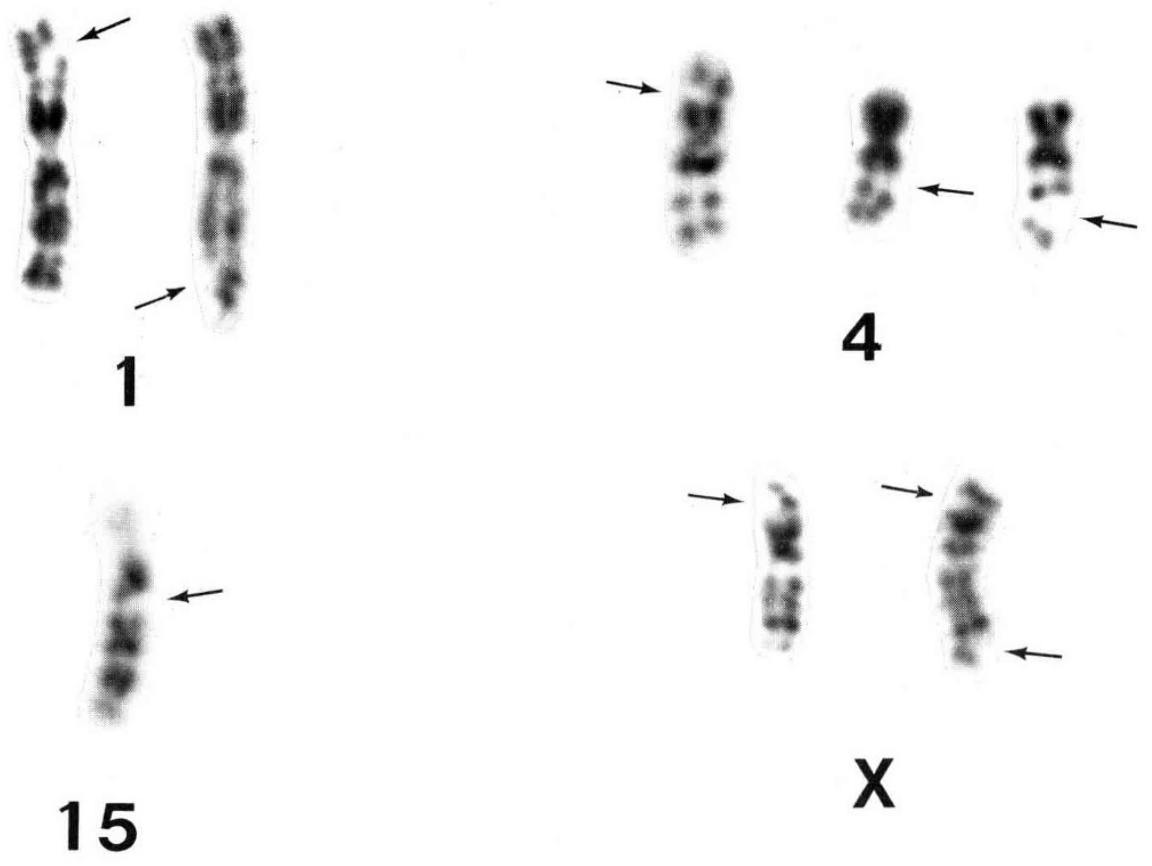

Fig 1. Localization of chromosomal breaks in Oryclolagus cuniculus L. Arrows indicate fragile sites localized to: 1 p32, 1q26, 4p14, 4q12, 4q14, 15q12, Xp14, Xp14 and Xq24.

Table II. Location and frequencies (\%) of putative fragile sites.

\begin{tabular}{lrrrrrr}
\hline $\begin{array}{c}\text { Animal/sex/ } \\
\text { treatment }\end{array}$ & \multicolumn{7}{c}{ Putative fragile sites } \\
\cline { 2 - 7 } & $1 p 32$ & $1 q 26$ & $4 p 14$ & $15 q 12$ & $X p 14$ & Xq24 \\
\hline A/F/APC & 28 & & 4 & 4 & 74 & 8 \\
A/F/AZA & 8 & 4 & 4 & & 24 & \\
B/F/APC & 18 & & 2 & & 16 & 2 \\
B/F/CAF & 8 & & & 6 & 20 & \\
C/M/APC & 16 & & & & 4 & \\
C/M/CAF & 10 & & 2 & 2 & 4 & \\
D/M/APC & 8 & & & & 26 & \\
D/M/CAF & 4 & & & 28 & & \\
\hline
\end{tabular}

A, B, C, D: animal number; F: female; M: male; APC: aphidicolin; AZA: $5^{\prime}$-cytidine; CAF: caffeine. 


\section{REFERENCES}

De Braeckeleer M (1987) Fragile sites and chromosomal structural rearrangements in human leukemia and cancer. Anticancer Res 7, 417-422

Hecht F, Ramesh KH, Lockwood DH (1990) A guide to fragile sites on human chromosomes. Cancer Genet Cytogenet 44, 37-45

Lubs HA (1969) A marker X chromosome. Am J Hum Genet 21, 231-244

Miro S, Clemente JC, Fuster C, Egozcue J (1987) Fragile sites, chromosome evolution and human neoplasia. Hum Genet 75, 345-349

Riggs PK, Chrisman CL (1989) Preliminary analysis of aphidicolin induced fragile sites in pig chromosomes. 6th North American Colloquium on Cytogenetics of Domestic Animals West Lafayette Indiana abstr 4

Rønne M (1983) Simultaneous R-banding and localization of dA-dT clusters in human chromosomes. Hereditas $98,241-248$

Rønne M (1984) Fluorouracil synchronization of human bone marrow cultures. In vitro induction of high resolution R-banding by simultaneous exposure to $5^{\prime}$-bromodeoxyuridine/Hoechst 33258. Anticancer Res 4, 279-282

Sutherland GR, Hecht F (1985) Fragile Sites on Human Chromosomes. Oxford University Press, New York

Yunis JJ, Soreng AL (1984) Constitutive fragile sites and cancer. Science 226, 1199-1204

Yunis JJ, Soreng AL, Bowe AE (1987) Fragile sites are the target of diverse mutagens and carcinogens. Oncogene 1, 59-69 